\title{
Eficiencia del anillado y profundidad de corte óptima para el control de dos especies leñosas exóticas invasoras del Chaco Serrano argentino
}

\author{
Bernasconi Salazar, J., Cora, A., Karlín, M. y González, L.
}

\begin{abstract}
RESUMEN
Las especies leñosas exóticas invasoras (ELEI) en sistemas montanos suelen tener alta demanda hídrica y sus tasas de producción de propágulos suelen ser elevadas. La supresión de individuos de ELEI mediante anillado provocaría una reducción en el input de propágulos, disminuyendo su tasa de expansión, y permitiendo mejorar las condiciones de competencia de la flora nativa. El objetivo del presente trabajo fue evaluar la eficiencia del anillado y la profundidad de corte óptima sobre la mortandad de Morus alba L. y Melia azedarach L. Con motosierra se realizaron uno o dos anillos sobre el fuste principal de individuos maduros de las especies mencionadas, y al cabo de dos años, se evaluó la sobrevida y el vigor de los individuos tratados. Para ambas especies el radio de corte óptimo se definió a través de un porcentaje del diámetro de fuste: los umbrales críticos variaron entre 11 y $13 \%$ del diámetro. Estos valores aseguran para $M$. alba y $M$. azedarach una eficiencia de tratamiento superior al $40 \%$. El anillado simple es el tratamiento más conveniente por su eficacia y por ser más económico con respecto al doble anillado.
\end{abstract}

Palabras clave: invasibilidad, sobrevida, Sensibilidad-Especificidad, KaplanMeier.

Bernasconi Salazar, J., Cora, A., Karlín, M. and González, L., 2018. Girdling efficiency and optimal cutting depth for the control of two invasive alien woody species in the Argentinian Chaco Serrano. Agriscientia 35 (2): 11-18

\section{SUMMARY}

Alien invasive woody species (AIWE) in mountain systems usually have high hydric demand and their rates of propagule production are generally elevated. The suppression of AIWE individuals through girdling should reduce propagule input, lowering their expansion rates and improving the conditions for native flora competition. The objective of this work was to evaluate the girdling efficiency and the optimal cutting depth over Morus alba L. and Melia azedarach L. mortality. With chainsaw, one or two girdles were made over the main stem in 
mature individuals of the mentioned species, and after two years, the survival and vigor of the treated individuals were evaluated. For both species the optimal cutting radio was defined through a percentage of the stem diameter: cutting critical threshold varied between 11 and $13 \%$ of the stem diameter. These values assure for $M$. alba and $M$. azedarach a treatment efficiency higher than $40 \%$. The single girdling is the most convenient treatment because of its efficiency and because it is cheaper than double girdling.

Key words: invasibility, survival, Sensitivity-Specificity, Kaplan-Meier

J. Bernasconi Salazar: Asociación Civil El Cuenco, Equipo Ambiental. Personería Jurídica Res. N³64 "A"/13. Tucumán 438, Pilar, CP 5972. Córdoba, Argentina. A. Cora: Asociación Civil El Cuenco, Equipo Ambiental. Instituto Nacional de Tecnología Agropecuaria, EEA Manfredi, Ruta 9 km 636, CP 5972. Córdoba, Argentina. M. Karlin: Asociación Civil El Cuenco, Equipo Ambiental. Universidad Nacional de Córdoba, Facultad de Ciencias Agropecuarias, Félix Marrone 746, Ciudad Universitaria, CC 509, CP 5000, Córdoba, Argentina. L. González: Universidad Nacional de Córdoba, Facultad de Ciencias Agropecuarias.Correspondencia a: ajijavier@gmail.com

\section{INTRODUCCIÓN}

Los ecosistemas pueden cambiar su fisonomía y funcionalidad en el tiempo en función a modificaciones ambientales, interacciones bióticas, invasión de especies exóticas y alteraciones en las frecuencias genotípicas. El ingreso de nuevas especies o genotipos puede ocurrir en forma natural o antrópica, pudiendo ser esta última accidental o intencional (Rejmánek, Richardson y Pyšek, 2013).

La invasión de especies exóticas puede provocar importantes cambios en el funcionamiento de los ecosistemas y en la oferta de servicios ecosistémicos, generando pérdidas importantes en términos económicos (Giorgis et al., 2011).

La tasa de invasión de especies exóticas depende directamente de la invasibilidad, es decir la susceptibilidad del ambiente a ser invadido; del input de propágulos de la taxa exótica, y del tratamiento e identidad de dicha taxa exótica (Rejmánek et al., 2013).

Los ecosistemas montanos presentan nichos invasibles dependiendo de las características de la vegetación, suelo, hidrología y microclima. Así, áreas donde circula agua libre (arroyos, ríos) son particularmente susceptibles de ser invadidas ya que muchas especies con alto potencial de invasión requieren altos contenidos de agua y pueden competir bien por otros recursos con las especies nativas (Tickner, Angold, Gurnell y Mountford, 2001; Schnitzler, Hale y Alsum et al., 2007). Las especies exóticas que aquí logran desarrollarse suelen generar grandes volúmenes de propágulos que facilitan y aceleran la expansión de sus poblaciones.

Según Giorgis et al. (2011), en las Sierras Centrales de Argentina la frecuencia de las especies leñosas exóticas invasoras (ELEI) aumentan según las siguientes características de hábitat: bajas altitudes, aumento de las pendientes de ladera, disminución en la distancia a rutas y asentamientos humanos.

Las ELEl que se desarrollan en las Sierras Chicas de Córdoba son particularmente demandantes en el consumo de agua de las freáticas (Zeballos et al., 2014) con relación a las especies nativas que se desarrollan en los mismos nichos. Esto provoca cambios en la dinámica hídrica de las cuencas hidrográficas, evidenciando efectos negativos sobre la disponibilidad, rendimiento y liberación de agua para uso humano, especialmente en ciclos secos (Mooney y Hobbs, 2000; Pejchar y Mooney, 2009; Jobbágy, Acosta y Nosetto, 2013).

Con relación al tratamiento de la taxa exótica, es de suponer que la supresión de individuos de ELEI provocará una reducción en el input 
de propágulos, reducirá su tasa de expansión (Rejmánek et al., 2013) y mejorará las condiciones de competencia de la flora nativa. Sin embargo, la supresión de estas especies es altamente costosa, especialmente si se han expandido en grandes áreas o si han generado bancos de semilla con alta densidad de propágulos (Richardson et al., 2007).

Áreas de alto valor de conservación, que han sido ya invadidas por estas especies, requieren de campañas de erradicación mediante tratamientos tales como anillado o aplicación de herbicidas, a pesar del costo de aplicación (Kleinbauer, Dullinger, Peterseil y Essl, 2010).

El anillado resulta ser un método interesante para el control de ELEl en áreas riparias ya que se evita la eliminación inmediata de los árboles en zonas susceptibles a erosión hídrica. El anillado provoca el debilitamiento y posterior muerte del individuo tratado, manteniendo una cobertura vegetal que protege el suelo del impacto directo de la gota de agua, cobertura que va disminuyendo gradualmente pero que favorecería la instalación de otras especies nativas (Loh y Daehler, 2008) reemplazando gradualmente a las ELEI.

Son escasos los estudios que analicen la efectividad del anillado como método para el control de especies exóticas leñosas (Goodland, Healy y Binggeli, 1998; Loh y Daehler, 2008) o como técnica silvicultural para el manejo de especies forestales (Brassiolo, Gómez, Senilliani y López, 2008; Améztegui, Aunós y Serrano, 2009). Asimismo, como método de bajo impacto ecológico en áreas protegidas, el anillado no ha sido estudiado como herramienta para contribuir a la conservación de la flora nativa o a la remediación de áreas invadidas por ELEI.

El objetivo del presente trabajo fue evaluar la eficiencia del anillado y la profundidad de corte óptima para el control de las especies leñosas exóticas Morus alba y Melia azedarach en una microcuenca del Chaco Serrano (Argentina).

Se pretende con este trabajo contribuir en la generación de información sobre técnicas de remediación de áreas invadidas por ELEI, así como evaluar metodologías estadísticas que no son aplicadas comúnmente en el ámbito de estudio de la remediación ecológica.

\section{MATERIALES Y MÉTODOS}

\section{Ubicación del área en estudio}

El estudio se efectuó en el sector oeste de la Reserva Natural de la Defensa La Calera, propio del cordón montañoso Sierras Chicas, que corresponde al distrito fitogeográfico Chaco Serrano, según la clasificación de Cabrera (1971).

El ensayo de anillado de especies forestales exóticas se realizó en la quebrada del Pumahuá, sobre una extensión de 1.500 m, coincidiendo con el área de influencia de la ribera del arroyo homónimo, abarcando aproximadamente un ancho de $40 \mathrm{~m}$. Está ubicada entre las cotas de $700 \mathrm{~m}$ s.n.m. $\left(31^{\circ} 24^{\prime} 11^{\prime \prime} \mathrm{S}, 64^{\circ} 24^{\prime} 11^{\prime \prime} \mathrm{O}\right)$ y $850 \mathrm{~m}$ s.n.m. $\left(31^{\circ}\right.$ $24^{\prime} 15^{\prime \prime} S, 64^{\circ} 24^{\prime} 59^{\prime \prime}$ O) sobre la ladera oriental del macizo de Sierras Chicas.

\section{Tratamientos aplicados}

Se aplicó la técnica del anillado, utilizando motosierras Stihl MS 271, efectuando uno $(n=49)$ o dos $(n=32)$ anillos sobre el fuste principal de árboles maduros de $M$. alba y $M$. azedarach de diámetro variable entre 11 y $86 \mathrm{~cm}$ (mediana: $31,5 \mathrm{~cm}$ ), a una altura de $1 \mathrm{~m}$ sobre el nivel del terreno. Las fechas de tratamiento fueron del 9 al 17 de octubre de 2013.

Los árboles tratados con anillado simple fueron 26 de M. alba y 23 de $M$. azedarach, los árboles tratados con anillado doble fueron 17 de M. alba y 15 de $M$. azedarach. Se tomaron al azar también 10 individuos de cada especie como testigos.

\section{Mediciones efectuadas}

Se midieron las profundidades de corte sobre cada uno de los anillos en cuatro puntos (norte, este, oeste y sur) a fin de calcular un promedio que fue utilizado para los análisis estadísticos. Esta variable fue denominada prof. En el caso de los anillados dobles, se utilizó para el análisis el valor más elevado entre los promedios de cada anillado.

Se midieron asimismo los valores de diámetro a la altura del pecho $(D A P)$ de cada uno de los individuos tratados.

Con las variables prof y DAP se construyó otra variable denominada \%corte que implica el porcentaje de diámetro cortado promedio en relación al diámetro del vástago.

La fecha de relevamiento del impacto de los tratamientos fue el 7 de octubre de 2015. En esta fecha se contabilizaron los individuos según la condición de la parte aérea (por encima de los anillos) como vivo (1) o muerto (0), si rebrotaron (1) o no (0) por debajo de los anillos, si formaron (1) o no (0) callos de cicatrización.

También se evaluó sobre los individuos vivos, el vigor de los individuos en forma subjetiva, 
aplicando una escala de 10 a 100, en categorías de diez en diez, según el porcentaje de foliosidad en el árbol, tomando como referencia los individuos testigo.

\section{Análisis estadístico}

Se realizaron dos regresiones logísticas para definir si las variables analizadas (regresoras), en este caso prof y \%corte, eran significativas como variables de análisis para los tratamientos de anillado. En ambos casos se evaluó como variable dependiente la situación binaria muerta/viva (0/1), aplicando como variables de clasificación la especie tratada (M. alba y $M$. azedarach) y el número de anillos (uno o dos anillos).

Se trataron los datos a través de un análisis de Sensibilidad-Especificidad (Fleming y Harrington, 1991), cuyas curvas, al cruzarse, definen un punto que corresponde al umbral crítico (Threshold). En este trabajo se evaluó la profundidad de corte más efectivo para el control de las especies exóticas forestales. La variable analizada fue binaria, es decir, muerta/viva (0/1) en función al porcentaje del diámetro de fuste cortado (\%corte) de la especie analizada, considerando como éxito del tratamiento los individuos muertos al momento de evaluación, luego de dos años desde el tratamiento.

Se realizaron también curvas de sobrevida de Kaplan-Meier (Efron, 1988), aunque adaptadas a la variable de interés que fue \%corte (de los dos cortes efectuados en los casos de anillado doble, se consideró el valor de \%corte mayor para el análisis) en lugar de tiempo. Aquí se analizaron en conjunto para cada especie los tratamientos de anillado simple y anillado doble a los fines comparativos $(P<0,05)$.

También se analizó mediante regresión logística la variable vigor de ambas especies en función de \%corte, usando como variables de clasificación la especie tratada y el número de anillos y considerando exitosos valores menores que la media.

Para la presencia/ausencia de callos o rebrote se calculó la probabilidad de ocurrencia de ambos fenómenos, comparando individuos de ambas especies tratadas.

Todos los análisis fueron realizados con el software estadístico InfoStat (Di Rienzo et al., 2017).

\section{RESULTADOS Y DISCUSIÓN}

La regresión logística con la variable prof no arrojó valores significativos $(P=0,4757)$ para ninguna de las combinaciones, es decir, anillado simple y anillado doble $(P=0,9483)$ para cada una de las especies ( $P=0,2992)$.

La regresión con la variable \%corte arrojó valores significativos $(P=0,0007)$, sin encontrar diferencias relevantes $(P<0,05)$ entre número de anillos $(P=0,6100)$ o especies $(P=0,1675)$; se obtuvo un valor de constante de la regresión de -2,90 (estimación de la ordenada al origen; $P=0,0009$ ) y un valor de la pendiente de la variable \%corte de 0,11 . Como la pendiente obtenida fue positiva, esta indica que la probabilidad que los individuos tratados resulten muertos aumenta con el porcentaje de corte. Para dicha variable la razón de chances de obtener individuos muertos es 1,12 veces mayor cuando el porcentaje de corte aumenta un $1 \%$.

De esta forma, para ambas especies (M. alba y $M$. azedarach) la efectividad del corte sobre la mortandad de los individuos tratados se definió a través de un porcentaje del diámetro de fuste (\%corte), no a través de una profundidad fija de corte. Esto podría deberse a que el efecto deletéreo de los cortes depende de que estos alcancen la zona del cámbium y alteren el flujo floemático, impidiendo la acumulación de fotosintatos en las raíces y corona (y probablemente, desabasteciendo de estos a las micorrizas de la rizósfera), responsables del rebrote de la temporada de crecimiento siguiente (Högberg et al., 2001).

El espesor del floema está íntimamente relacionado con el diámetro total de los vástagos de los árboles (De Schepper, van Dusschoten, Copini, Jahnke y Steppe, 2012) por lo que si el principio del tratamiento es cortar la circulación floemática en su totalidad, este corte resulta ser función del diámetro del vástago.

De acuerdo a las curvas de Sensibilidad-Especificidad de la Figura 1 los umbrales críticos (Threshold) variaron entre 22,7 y $24,5 \%$ del diámetro de fuste, lo que significa que el tratamiento comenzó a ser efectivo a partir de un radio de corte del 11 a $13 \%$ sobre el diámetro del fuste a cortar (es decir, si por ejemplo el fuste tenía $20 \mathrm{~cm}$, el tratamiento tuvo efecto significativo realizando cortes de un radio de 2,2 a 2,6 cm de profundidad).

De los testigos sin tratamiento, sobrevivió el $100 \%$ hasta el momento de las mediciones. La técnica del anillado simple frente al anillado doble no arrojó diferencias significativas $(P<0,05)$ en cuanto a la eficacia del tratamiento para ninguna de las especies analizadas (Figura 2).

Para M. alba puede apreciarse en la Figura 2a que, si bien no existieron diferencias estadísticamente significativas $(P<0,05)$ entre anillado simple y doble, el último promovió una mayor mortandad 


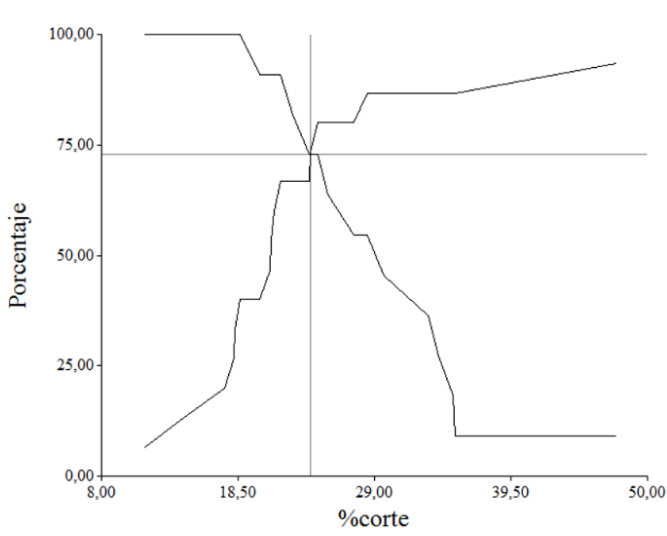

C

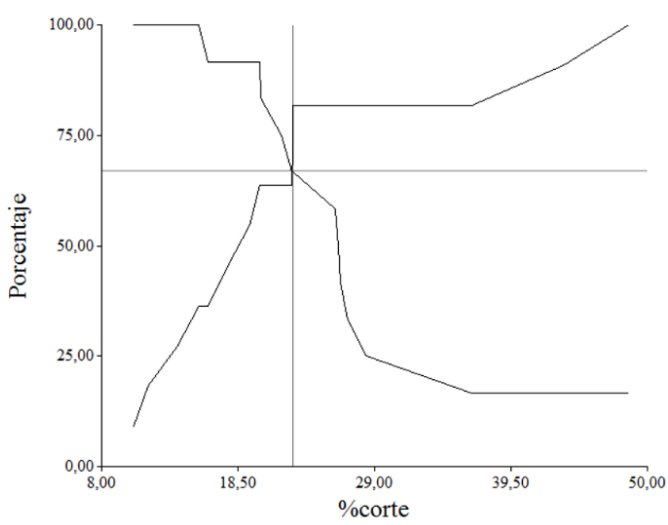

$b$

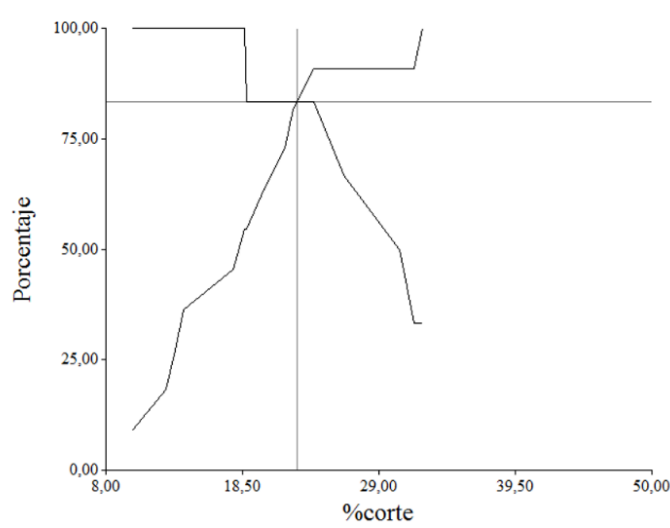

$d$

M. azedarach, anillo doble

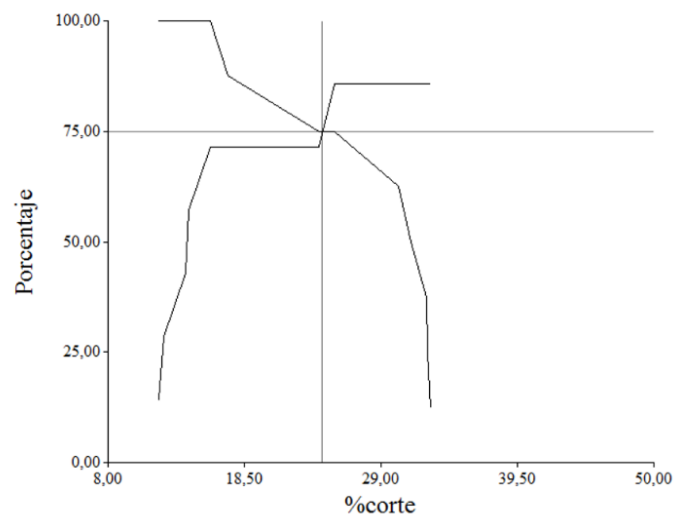

Figura 1: Curvas de Sensibilidad-Especificidad para a) Morus alba, anillo simple (Threshold: 24,1 \%; $n=26$ ); b) Morus alba, anillo doble (Threshold: 22,7 \%; n=17); c) Melia azedarach, anillo simple (Threshold: 22,7 \%; $n=23$ ); d) Melia azedarach, anillo doble (Threshold: $24,5 \% ; n=15)$

$a$

M. alba

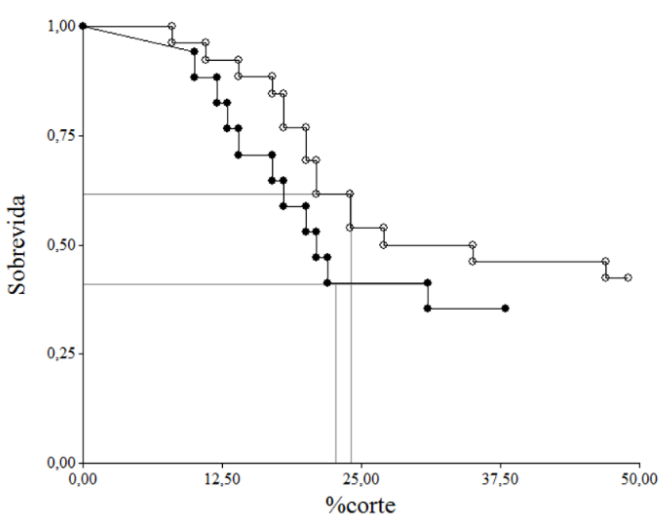

$b$

M. azederach

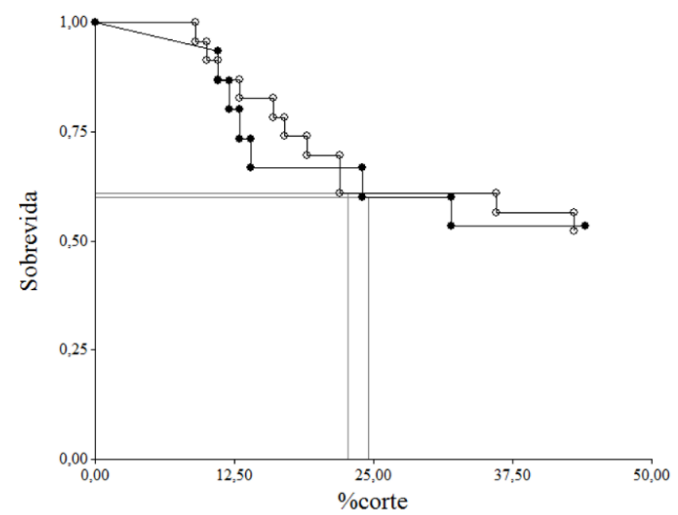

Figura 2: Curva de sobrevida en función al \%corte para tratamiento de anillado simple (círculos vacíos) y anillado doble (círculos llenos), para a) Morus alba (Chi-cuadrado para el log Rank test $=0,711 ; P=0,399$, no significativo al $P_{\text {valor }}=0,05$; Threshold: $24,1 \%$ y $22,7 \%$ para anillado simple y doble, respectivamente) y b) Melia azedarach (Chi-cuadrado para el log Rank test $=0,004 ; P=0,948$, no significativo al $P_{\text {valor }}=0,05$; Threshold: $22,7 \%$ y $24,5 \%$ para anillado simple y doble, respectivamente). 
de individuos a menor \%corte y además logró una mortandad (nótese que mortandad es igual a 100 menos la sobrevida, si se observa la Figura 2) marginalmente superior al $50 \%$ al alcanzar el umbral crítico de corte $(22,7 \%)$, mientras que el umbral crítico para el anillado simple provocó una mortandad menor al $50 \%$. El anillado simple alcanzó una mortandad del $50 \%$ por encima del $27 \%$ del \%corte.

La eficiencia del anillado para $M$. azedarach (Figura 2b) resultó ser menor que para M. alba, no superando el $50 \%$ de mortandad en ninguno de los dos casos. Las curvas para anillado simple y doble difunden en forma más pareja que en $M$. alba, obteniendo 39 y $40 \%$ de mortandad en sus umbrales críticos $(22,7$ y $24,5 \%$ del \%corte, respectivamente).

La variable \%corte, dependiente del diámetro del vástago, define umbrales para tratamientos de anillado simple y doble y para ambas especies que son muy cercanos entre sí, lo que facilita la recomendación de la aplicación de intensidades de corte para estas leñosas. Fredericksen, Contreras y Pariona (2001) recomiendan para tratamientos de aclareo en plantaciones forestales cortes de $3 \mathrm{~cm}$ de radio en árboles no deseados, complementado con aplicaciones de herbicidas. El problema de complementar el tratamiento con la aplicación de herbicidas radica en que su aplicación puede que no esté permitida en algunas áreas protegidas, tales como la de la Reserva Natural Militar La Calera. Según Frederiksen et al. (2001) los árboles anillados sin aplicárseles herbicida pueden demorar varios años en morir y, en muchos casos, sus tejidos pueden cicatrizar sobre el corte de anillamiento, evitando así la muerte.

Cuando los árboles anillados mueren, sus ramas se pudren y caen durante un período prolongado de tiempo y su fuste se puede mantener en pie por varios años. La disminución gradual de la cobertura de hojas y ramas de los competidores anillados también brinda un período de transición, en el cual los árboles de sustitución nativos se pueden ajustar al aumento de luz (Frederiksen et al., 2001; Loh y Daehler, 2008) y los árboles tratados pueden proteger frente a procesos de erosión en sitios de pendientes elevadas.

La efectividad del tratamiento indica en este estudio que a los dos años de efectuado se podría esperar alrededor de un $50 \%$ de respuesta en relación a la mortandad. Porcentajes similares también fueron encontrados por Brassiolo et al. (2008) en tratamientos con anillado triple sobre Gleditsia amorphoides (Griseb.) Taub., Senegalia praecox (Griseb.) Seigler \& Ebinger, pero ambos evaluados al año del tratamiento, y Cordia americana (L.) Gottschling y J.S. Mill., evaluado a los tres años del tratamiento.

En experimentos efectuados en dos bosques de Bolivia se observó que el anillado después de un solo año, sin aplicación de herbicida, causó la muerte de 10 a 12 \% de los árboles tratados (Fredericksen et al., 2001), aunque los autores no aclaran qué especies fueron tratadas.

La regresión logística para la variable categórica vigor definió una relación significativa $(P=0,0139)$ entre el porcentaje del área basal cortada (\%corte) y la pérdida de vigor del árbol, sin encontrar diferencias entre el número de anillos $(P=0,2047)$ o especie tratada $(P=0,6258)$. La ordenada al origen fue de $-0,97(P=0,1738)$ y la pendiente de la variable \%corte de 0,07. La razón de chances para dicha variable fue 1,07 .

El vigor observado para ambas especies evaluadas fue dependiente de la intensidad del corte efectuado, pero también del tamaño del diámetro de fuste, variable implícita en \%corte. La relación entre el vigor de los individuos y el diámetro de fuste se explica en relación a la cantidad de reservas disponibles en los tejidos aéreos. Ante la imposibilidad de destinar reservas desde las raíces a los órganos aéreos en las épocas de reactivación de los mecanismos fisiológicos de rebrote o de formación de propágulos, la planta hidroliza almidón de los tejidos aéreos por lo que no muere inmediatamente y el proceso es más lento a mayor tamaño de la planta (Améztegui et al., 2009).

Los signos de rebrote no siempre se encontraron en todos los arboles tratados, pero en los que se observó fue en la parte inferior del corte y en el anillo más próximo al suelo, en el caso del doble anillado.

No se evidenció una relación significativa entre la formación de callo y la sobrevida de los individuos; las probabilidades de encontrar individuos sobrevivientes con callo fueron del 34,6 y 38,9 \% para $M$. alba y $M$. azedarach respectivamente. La formación de callo implica la proliferación de células pluripotentes capaces de diferenciarse posteriormente (aunque no inmediatamente) en tejido xilemático o floemático, pero anteriormente a ello no existe conectividad entre ambas porciones de la planta. Esa diferenciación dependerá de la producción de auxinas y citoquininas promovidas por la herida producida a la planta, pero también dependerá de la presencia y acción de genes inhibidores o retardadores del flujo, aumento de la concentración y respuesta de las auxinas sobre la diferenciación de las células del callo (Melnyk, Schuster, Leyser y Meyerowitz, 2015). 
Por otro lado, tampoco existe una clara relación entre los individuos muertos y la formación de rebrote por debajo de los anillos; las probabilidades encontradas fueron de 54,7 y $26,3 \%$ para $M$. alba y $M$. azedarach respectivamente. La formación de rebrotes por debajo del corte puede manifestarse como un buen indicador de que efectivamente la conectividad entre raíz y copa se ha suprimido. Sin embargo, es probable que aquellos brotes generados en la zona del corte formen un puente de conducción entre ambas partes de la planta si dicho brote alcanza a cicatrizar con la porción superior de la planta.

\section{CONCLUSIONES}

El porcentaje de corte en función al diámetro del vástago resultó ser un índice más confiable que los valores fijos de corte para definir la efectividad de los tratamientos de anillado.

El anillado simple es el tratamiento más conveniente por su eficacia, y por ser más económico con respecto al doble anillado.

No existe relación entre la formación de tejido cicatrizante (callo) y la sobrevida de árbol, así como tampoco hay relación entre la mortandad y la aparición de rebrotes por debajo del anillado.

Se recomienda en todos los casos la aplicación de radios de corte superiores al $13 \%$ del diámetro del vástago a tratar. Estos valores aseguran para $M$. alba y $M$. azedarach mortandades superiores al 40 \% sin aplicación complementaria de productos fitosanitarios.

\section{AGRADECIMIENTOS}

Los autores agradecen a la Fundación AVINA por el financiamiento efectuado al proyecto " $E$ I manejo como herramienta para la recuperación de cuencas". Los autores agradecen también a Juan Bernasconi por su colaboración en tareas de campo, y a Margot Tablada y Pablo Paccioretti por sus aportes en los análisis estadísticos.

\section{BIBLIOGRAFÍA}

Améztegui, A., Aunós, A., y Serrano, L. (2009). El anillado como técnica de desvitalización de hayas en el Valle de Arán (Lleida). V Congreso Forestal Español, Ávila, España. 12 p.

Brassiolo, M. M., Gómez, C., Senilliani, M. G., y López, C. (2008). Mortalidad selectiva inducida para raleos en bosques nativos. Quebracho - Revista de Ciencias Forestales, 16, 94-101.

Cabrera, A. L. (1971). Fitogeografía de la República Argentina. Boletín de la Sociedad Argentina de Botánica, 14, 1-42.

De Schepper, V., van Dusschoten, D., Copini, P., Jahnke, S., y Steppe, K. (2012). MRI links stem water content to stem diameter variations in transpiring trees. Journal of Experimental Botany, 63(7), 2645-2653. DOI: 10.1093/jxb/err445.

Di Rienzo, J., Casanoves, F., González, L., Tablada, M., Robledo C., y Balzarini, M. (2017). Infostat. Software estadístico. Córdoba, Argentina. Facultad de Ciencias Agropecuarias, Universidad Nacional de Córdoba.

Efron, B. (1988). Logistic regression, survival analysis, and the Kaplan-Meier curve. Journal of the American Statistical Association, 83(402), 414-425.

Fleming, T. R., y Harrington, D. P. (1991). Counting processes and survival analysis. Nueva York, Estados Unidos: John Wiley y Sons, Inc.

Fredericksen, T., Contreras F., y Pariona, W. (2001). Guía de silvicultura para bosques tropicales de Bolivia. Santa Cruz, Bolivia: Proyecto BOLFOR.

Giorgis, M. A., Tecco, P. A., Cingolani, A. M., Renison, D., Marcora P., y Paiaro, V. (2011). Factors associated with woody alien species distribution in a newly invaded mountain system of central Argentina. Biological Invasions, 13(6), 1423-1434. DOI: 10.1007/s10530-0109900-y.

Goodland, T. C. R., Healy J. R., y Binggeli, P. (1988). Control and management of invasive alien woody plants in the tropics. Publication Number 14, School of Agricultural and Forest Sciences, University of Wales.

Högberg, P., Nordgren, A., Buchmann, N., Taylor, A. F. S., Ekblad, A., Högberg, M. N., Nyberg, G., Ottosson-Lofvenius, M., y Read, D. J. (2001). Large-scale forest girdling shows that current photosynthesis drives soil respiration. Nature, 411, 789-792. DOI: 10.1038/35081058

Jobbágy, E. G., Acosta, A. M., y Nosetto, M. D. (2013). Rendimiento hídrico en cuencas primarias bajo pastizales y plantaciones de pino de las sierras de Córdoba (Argentina). Ecología Austral, 23(2), 87-96.

Kleinbauer, I., Dullinger, S., Peterseil, J., y Essl, F. (2010). Climate change might drive the invasive tree Robinia pseudacacia into nature reserves and endangered habitats. Biological Conservation, 143(2), 382-390. DOI:10.1016/j.biocon.2009.10.024.

Loh, R. K., y Daehler, C. C. (2008). Influence of woody invader control methods and seed availability on native and invasive species establishment in a Hawaiian forest. Biological Invasions, 10(6), 805-819. DOI 10.1007/s10530-008-9237-y. 
Melnyk, C. W., Schuster, C., Leyser, O., y Meyerowitz, E. M. (2015). A developmental framework for graft formation and vascular reconnection in Arabidopsis thaliana. Current Biology, 25(10), 1306-1318. DOI: 10.1016/j.cub.2015.03.032.

Mooney, H. A., y Hobbs, R. J. (2000). Invasive species in a changing world. Washington, DC., Estados Unidos: Island Press.

Pejchar L., y Mooney, H. A. (2009). Invasive species, ecosystem services and human well-being. Trends in Ecology \& Evolution, 24(9), 497-504. DOI:10.1016/j. tree.2009.03.016.

Rejmánek, M., Richardson, D. M., y Pyšek, P. (2013). Plant invasions and invasibility of plant communities. En Van Maarel, E., y Franklin, J. (Eds.). Vegetation Ecology. Second Edition. Wiley, pp. 387-424.
Richardson, D. M., Holmes, P. M., Esler, K. J., Galatowitsch, S. M., Stromberg, J. C., Kirkman, S. P., Pyšek, P., y Hobbs, R. J. (2007). Riparian vegetation: degradation, alien plant invasions, and restoration prospects. Diversity and Distributions, 13(1), 126-139. DOI: 10.1111/j.1472-4642.2006.00314.x.

Schnitzler, A., Hale, B. W., y Alsum, E. M. (2007). Examining native and exotic species diversity in European riparian forests. Biological Conservation, 138(1), 146156. DOI:10.1016/j.biocon.2007.04.010.

Tickner, D. P., Angold, P. G., Gurnell, A. M., y Mountford, J. O. (2001). Riparian plant invasions: hydrogeomorphological control and ecological impacts. Progress in Physical Geography, 25(1), 22-52.

Zeballos, S. R., Giorgis, M. A., Cingolani, A. M., Cabido, M., Whitworth Hulse, J. I., y Gurvich, D. E. (2014). Do alien and native tree species from Central Argentina differ in their water transport strategy? Austral Ecology, 39(8), 984-991. DOI:10.1111/aec.12171. 